\title{
Double Row Absorbable Anchor Bridge Suture Versus Hollow Nails for Treating Acute Posterior Cruciate Ligament Avulsion Fracture
}

gan zhang ( $\square$ zhanggan10282715@163.com )

the 901th Hospital of Chinese People's Liberation Army

Lu Wu

The 901 th Hospital of the JLSF

Qianyong Chen

The 901 th Hospital of the JLSF

Research article

Keywords: Posterior cruciate ligament,Fracture,Double row absorbable anchor,Bridge suture

Posted Date: September 28th, 2021

DOl: https://doi.org/10.21203/rs.3.rs-919259/v1

License: () (i) This work is licensed under a Creative Commons Attribution 4.0 International License. Read

Full License 


\section{Abstract}

Objective To compare the outcomes of open reduction through double row absorbable anchor bridge suture with hollow nails for acute posterior cruciate ligament $(\mathrm{PCL})$ avulsion fracture. Methods From May 2015 to May 2019, 35 patients with acute PCL avulsion fractures were treated by open reduction through double row absorbable anchor bridge suture or hollow nails. There were 15 cases in double row absorbable anchor group and 20 cases in hollow nails group. The operation time, incision length, postoperative ambulation time, hospitalization expenses, healing time of fracture and Lysholm score of patients at the last follow-up were compared between the two groups. Result All the patients in both group had operation perfromed smoothly without serious complication. The operation time in double row absorbable anchor group was $(77.33 \pm 9.88)$ min which was longer compared to $(59.75 \pm 7.86) \mathrm{min}$ in the hollow nails group ( $\mathrm{p} \mathbb{}$ 0.05). The hospitalization expenses indouble row absorbable anchor group was (28132 \pm 2096$)$ yuan which was higher compared to $(15904 \pm 1113)$ yuan in the hollow nails group $(\mathrm{p} \otimes 0.05)$. The postoperative ambulation time in double row absorbable anchor group was $(2.07 \pm 0.70) \mathrm{d}$ which was shorter compared to $(3.80 \pm 1.64) d$ in the hollow nails group ( $p \otimes 0.05)$. There were no significant difference between the two

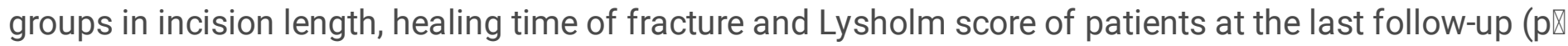
0.05). Conclusion Both open reduction through double row absorbable anchor bridge suture and hollow nails for acute PCL avulsion fracture have good clinical result. Double row absorbable anchor bridge suture had advantage of reliable fixation and shorter postoperative ambulation time incision length, however, hollow nails fixation had advantages of less operation time and lower hospitalization expenses.

\section{Introduction}

Acute posterior cruciate ligament (PCL)avulsion fracture is rare in clinic, although in countries like China and India in which motorcycle accidents are frequent[1].Inappropriate treatment of PCL avulsion fracture leads to an incompetent PCL which can result in knee instability and osteoarthritis.[2] Many authors recommend operative treatment of a PCL avulsion fracture is regarded if the bone fragment emerges above the joint line on lateral knee radiographs and the bone fragment has been displaced more than 5 $\mathrm{mm}$ on magnetic resonance imaging[3-4]Many operative methods (open surgical approaches and arthroscopic techniques) for treatment are reported, but there is no consensus regarding the optimal surgical approach for these injuries[5]. The optimal surgical treatment of isolated PCL tibial avulsion fractures remains controversial.The optimal fixation method is another controversial issue. There is a wide variety of materials for internal fixation, including hollow screws, wire, absorbable suture anchors, and toothed plates[6-8].

The aim of this study was to compare the clinical outcomes of acute posterior cruciate ligament avulsion fractures treated with double row absorbable anchor bridge suture versus hollow nails.

\section{Materials And Methods}

A total of 35 patients with posterior cruciate ligament avulsion fractures from May 2015 to May 2019 were researched, Some patients ( group A) received double row absorbable anchor bridge suture, and the others 
( group B ) received hollow nails. The fracture pattern was not considered in the selection of different fixation methods. There were no differences in age, sex, or body mass index between the two groups (Table 1). The inclusion criterion for the study was a displaced PCL tibial avulsion fracture (displacement of the bony fragment $>5 \mathrm{~mm}$ ) with an obvious positive posterior draw test. Patients with a combined tibial plateau fracture and large fragment of PCL tibial avulsion fracture, distal femur fracture, or neurovascular injury were excluded. meanwhile区patients with no displacement or little displacement (less than $5 \mathrm{~mm}$ ) fracture were excluded.

A prospective study was performed to assess patients who received double row absorbable anchor bridge suture, and the others received hollow nails.

\section{Surgical procedure}

\section{The double row absorbable anchor bridge suture group》}

Spinal anesthesia was administered. A conventional prone position with the knee in the 20 to 30 degrees flexion and a thigh pneumatic tourniquet around the thigh were used. Inverted "L" shaped incision was used in posterior knee joint. Skin vertical incision was made over the posterior aspect of the knee with optional horizontal extension. We performed the posterior approach described by Burks and Schafer [9]. Blunt dissection was done between the medial head of the gastrocnemius and semitendinosus down to the joint capsule. A longitudinal incision was made on the posterior articular capsule of the knee to expose the PLC and bone fragment. After the debridement of fracture loaction, an internal anchor (Johnson \& Johnson,USA) was inserted into central region of the fracture loaction. 4 sutures based anchor were passed through the osteoteninous junction of fracture location, respectively. The 4 sutures were distributed at a similar distance. The pilot hole for the footprint anchor was made about $1.5 \mathrm{~cm}$ inferior to the distal edge of the fracture bed, and the anchor was inserted along with four sutures into the hole keeping the sutures manually tight, thereby giving the adequate compressive force of the fragment across the fracture bed. The suture was released, and the anchor was inserted into the laser line at the level of the bone surface by turning the handle clockwise and slightly pressing downward. The posterior capsule and the wound was sutured in layer by layer. As shown in Figure 1. .typical cases are shown in Figures 2】

\section{The hollow nail group》}

Spinal anesthesia was administered. A conventional prone position with the knee in the 20 to 30 degrees flexion and a thigh pneumatic tourniquet were used. Inverted "L" shaped incision was used in posterior knee joint. Skin vertical incision was made over the posterior aspect of the knee with optional horizontal extension. We performed the posterior approach described by Burks and Schafer [9]. Blunt dissection was done between the medial head of the gastrocnemius and semitendinosus down to the joint capsule. $A$ longitudinal incision was made on the posterior articular capsule of the knee to expose the PLC and bone fragment. After the bone fragment was reduced, a 1-mm Kirschner wire was drilled from the center of the 
bone fragment to Anterior knee joint for temporary fixation. Next, the fragment was stabilized with hollow nails with a diameter of $4.0 \mathrm{~mm}$. One or two nails with length 50 or $55 \mathrm{~mm}$ were used. After fixation, the plain radiograph was used to check the screw position. If considered to be satisfactory, the tourniquet was defflated. The posterior capsule and the wound was sutured in layer by layer.囚typical cases are shown in Figures 3区

\section{Postoperative Rehabilitation}

Postoperatively, the limb was kept immobilized using a long knee brace for 6 weeks. Quadriceps strengthening was started from the 2nd postoperative day. Passive knee bending was started after 2 weeks and active knee mobilization was started 3 weeks after surgery. Partial weight bearing activities were performed after 6 weeks and full weight bearing were performed after 12 weeks while the brace was also discarded. Return to heavy activities, such as running and sports, were allowed only after 6-9 months.

\section{Statistical Analysis}

The operative time, incision length, postoperative ambulation time, time of hospitalization, hospitalization cost, fracture healing time, Lysholm score at the last follow-up and postoperative complications were collected in the two groups. Comparisons between groups were done by one-way analysis of variance (ANOVA). Statistical analyses were performed with The SPSS 19.0. The differences were considered as statistical significance at the level of $P<0.05$.

\section{Result}

Patients in both groups were successfully operated without serious complications such as vascular and nerve injury. The perioperative data of the two groups were shown in Table 1. The operation time of the double row absorbable anchor bridge suture group was longer than that of the hollow nails group, the postoperative ambulation time was shorter than that of the hollow nails group, and the hospitalization cost of the double row absorbable anchor bridge suture group was higher than that of the hollow nails group, and the differences were statistically significant $(P<0.01)$. There was no statistical significance in the length of incision and time of hospitalization between the two groups $(P>0.05)$, as shown in Table 2 . 
Table 1 Patient Demographics

\begin{tabular}{lcccccc}
\hline \multirow{2}{*}{ Group } & $\mathrm{n}$ & \multicolumn{2}{c}{ Sex } & \multicolumn{2}{c}{ Lateralization } & \multirow{2}{*}{ Age(year) } \\
\cline { 3 - 6 } & & Man & Feman & Left & Right & \\
\hline GroupA & 15 & 8 & 7 & 10 & 5 & $41.00 \pm 14.17$ \\
Group B & 20 & 11 & 9 & 12 & 8 & $35.65 \pm 10.25$ \\
$\chi^{2 / t \text { value }}$ & & & 0.01 & 2.44 & 1.30 \\
P value & & & 0.92 & \multicolumn{2}{c}{0.12} & 0.20 \\
\hline
\end{tabular}

\begin{tabular}{|c|c|c|c|c|c|c|}
\hline \multicolumn{7}{|c|}{ Table 2} \\
\hline group & $\mathrm{n}$ & 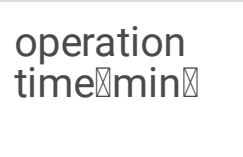 & $\begin{array}{l}\text { length of } \\
\text { incision } \\
\text { 『cm } \rrbracket\end{array}$ & $\begin{array}{l}\text { postoperative } \\
\text { ambulation time } \\
\rrbracket \mathrm{d} \rrbracket\end{array}$ & 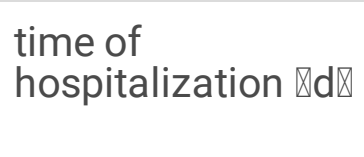 & $\begin{array}{l}\text { hospitalization } \\
\text { cost } \llbracket y u a n \rrbracket\end{array}$ \\
\hline A & 15 & $77.33 \pm 9.80$ & $6.93 \pm 1.62$ & $2.07 \pm 0.70$ & $13.13 \pm 4.19$ & $28132 \pm 2096$ \\
\hline B & 20 & $59.75 \pm 7.86$ & $6.80 \pm 0.52$ & $3.80 \pm 1.64$ & $13.70 \pm 4.60$ & $15904 \pm 1113$ \\
\hline $\mathrm{t}$ value & & 5.89 & 0.31 & -4.23 & -0.37 & 15.46 \\
\hline$P$ vakue & & $\nabla 0.01$ & 0.76 & $\nabla 0.01$ & 0.71 & $\varangle 0.01$ \\
\hline
\end{tabular}

Patients in both groups were followed up for 8-15 months, with an average of $(11.14 \pm 2.10)$ months. During the follow-up, there was no joint infection or internal fixation failure in both groups. At the final follow-up, Lysholm score was $(93.93 \pm 2.46)$ in the double row absorbable anchor bridge suture group and (92.65 \pm 2.30$)$ in the hollow nails group, and no significant difference was obtained between the two groups $(\mathrm{t}=1.59, \mathrm{P}=0.12)$.

At the last follow-up, all the 15 patients in the double row absorbable anchor bridge suture group walked normally without claudication or obvious limitation of knee flexion and extension, and all the patients recovered their movement and labor ability before injury. Among the 20 patients in the the hollow nails group, 2 had mild claudication and 1 had mild limitation of flexion movement. One case did not reach the level of working ability of injured sports. There was no obvious quadriceps femoris atrophy in 41 patients in both groups, and the results of the back drawer test were negative. One patient complained of residual pain and swelling in the joint. One of the patients had superfificial wound infection, which was treated with oral antibiotics and regular dressings. No other complications were observed.

\section{Image evaluation}

Postoperative imaging showed that anatomical reduction was achieved in 15 cases of the double row absorbable anchor bridge suture group and 19 cases of the hollow nails group. Imaging fracture healing time was $(2.40 \pm 0.51)$ months in the double row absorbable anchor bridge suture group and $(2.50 \pm 0.51)$ 
months in the hollow nails group, and there was no significant difference between the two groups $(t=-0.57$, $\mathrm{P}=0.57)$.

\section{Discuss}

Conservative treatment of PCL tibial avulsed fracture may lead to relaxation of secondary knee stability factors, knee pain, vertical and rotational instability in the short term, and osteoarthritis in the long term [2].Zhao et al. [4] believed that surgical treatment should be considered when avulsion fracture fragment shifted $>5 \mathrm{~mm}$. Therefore, for acute displaced PCL tibial avulsion fracture, early surgery is advocated to restore fracture reduction and PCL tension, and to reconstruct the stability of knee joint.

Common surgical approaches for open surgery include the a posterior approach[10], modifified posterior approach [11], the modified Burks-Schaffer approach [9], and so on. Modified Burks-Schaffer approach was adopted in all cases in this study, which was characterized by simple anatomy, clear exposure, simple operation, high safety and almost no possibility of injury to the vascular and nerve bundles behind the knee joint.

Clinically, there are many kinds of internal fixation materials for the treatment of PCL tibial avulsed fracture, and hollow nails is widely used because it can provide enough stability. In our study, all patients in the hollow nails group were fixed with two hollow nails, which provided good compression effect and excellent anti-rotation ability. Early functional exercises can be done, and all patients obtained good knee function after surgery. However, multiple hollow nails fixation requires avulsion of the fracture block and a large piece of intact bone to be inserted into the screw, which is difficult to achieve in the treatment of comminuted fractures. Therefore, as to comminuted fractures, threaded anchors are a better choice. The threaded anchor can penetrate into the bone to provide good holding power, and the high-strength suture ensures the firm fixation of the fracture block [10].Compared with hollow nails fixation, this suture method has several advantages :(1) Anatomic reduction can be achieved no matter what fracture shape and size of fracture. (2) Bridge suture technology can provide good compression effect on avulsed fracture, and good bone contact at the broken end which is conducive to the healing of fracture [12].(3) The anchor offer strong purchase and sutures resist strong tension, which result reliabe fixation. (4) The absorbable anchor is biodegradable and does not need to be removed by a second operation, which can release the patients' physical discomfort caused by the retention of the implant. Above advantages double row anchor group of patients can earlier on. Of course, double row absorbable anchor bridge suture also has many disadvantages: (1) longer operation time, two double-row anchor sutures need to be placed intraoperatively, and the four-ply suture lines should be properly spaced and evenly distributed, which is inconvenient to operate in a narrow space. (2) higher treatment cost. the absorbable anchors used in the double row absorbable anchor bridge suture group in this study were produced by Johnson \& Johnson Company. The overall treatment cost $(28132 \pm 2096$ yuan) was much higher than that of the hollow nails group (15,904 \pm 1113 yuan). (3) External anchors fixed to the posterior tibial cortex may cause fracture during placement. 
In conclusion, both open reduction and double-row absorbable anchor bridging-type suture and cannulated screw fixation can achieve good surgical results in the treatment of avulsion fractures of posterior cruciate ligament at tibial insertion. The double row absorbable anchor bridge suture provides a firm fixation, less time in the ground, longer operation time and higher hospitalization costs.

\section{Abbreviations}

$\mathrm{PCL}=$ posterior cruciate ligament.

\section{Declarations}

\section{Ethics approval and consent to participate}

Each subject had signed the informed consent before participating in our study. This study was approved by the ethics committee of the 901th Hospital of the Joint Logistics Support Force of the Chinese People's Liberation Army

\section{Consent for publication}

I declare that I have obtained explicit permission from all co-authors to submit this paper in its current form

\section{Availability of data and materials}

The datasets generated and/or analyzed during the current study are not publicly available due to statutory provisions regarding data and privacy protection but are available from the corresponding author on reasonable request.

\section{Competing interests}

The authors declared no conflicts of interest.

\section{Funding}

None

\section{Authors' contributions}


Gan Zhang, and Lu WU were involved in the conception and design of the study; the collection, assembly, analysis and interpretation of the data; and in drafting of the article. The others provided statistical expertise and contributed to the final approval of the article, provision of study materials, technical and logistical support.

\section{Acknowledgements}

Not applicable.

\section{Authors' information (optional)}

Gan Zhang

Orthopaedics

The 901th Hospital of the Joint Logistics Support Force of the Chinese People's Liberation Army,Hefei 230031, China

E-mail:zhanggan10282715@163.com

Lu Wu

Orthopaedics

The 901th Hospital of the Joint Logistics Support Force of the Chinese People's Liberation Army,Hefei 230031, China

E-mail:gyk966250@163.com

\section{References}

1. Abdelbadie A, Arafa MS.Treatment of posterior cruciate ligament tibial avulsion by a minimallyinvasive open posterior approach.Injury,2017,48(7):1644-1649.

2. Katsman A, Strauss EJ, Campbell KA, Alaia MJ. Posterior cruciate ligament avulsion fractures. Curr Rev Musculoskelet Med. 2018;11(03):503-9.

3. Gui J, Wang L, Jiang Y, et al. Single-tunnel suture fixation of posterior cruciate ligament avulsion fracture. Arthroscopy. 2009;25(1):78-85.16.

4. Zhao J, He Y, Wang J. Arthroscopic treatment of acute tibial avulsion fracture of the posterior cruciate ligament with suture fixation technique through Y-shaped bone tunnels. Arthroscopy. 2006;22(2):17281. 
5. Montgomery SR, Johnson JS, McAllister DR, Petrigliano FA. Surgical management of PCL injuries: indications, techniques, and outcomes. Curr Rev Musculoskelet Med. 2013;6(02):115-23.

6. Katsman A, Strauss EJ, Campbell KA, Alaia MJ. Posterior cruciate ligament avulsion fractures. Curr Rev Musculoskelet Med. 2018;11(03):503-9.

7. Lee KW, Yang DS, Lee GS, Choy WS. Suture bridge fifixation technique for posterior cruciate ligament avulsion fracture. Clin Orthop Surg. 2015;7(04):505-8.

8. Chen W, Luo W, Chen Z, Jiang Y. Treatment of posterior cruciate ligament avulsion fractures of the tibia using a toothed plate and hollow lag screw. Singapore Med J. 2016;57(1):39-44.

9. Burks RT, Schaffer JJ. A simplified approach to the tibial attachment of the posterior cruciate ligament. Clin Orthop Relat Res,1990(254):216-219.

10. Abbott LT, Saunders J, Bost FC, Anderson CE. Injuries to the ligaments of the knee joint. J Bone Joint Surg. 1944;26:503-21.

11. Nicandri GT, Klineberg EO, Wahl CJ, Mills WJ. Treatment of posterior cruciate ligament tibial avulsion fractures through a modifified open posterior approach: Operative technique and 12-to 48-month outcomes. J Orthop Trauma. 2008;22(5):317-24.

12. Xu XZhang,GuopingCai,Jie,Kun Wang. A minimally invasive postero-medial approach with suture anchors for isolated tibial avulsion fracture of the posterior cruciate ligament.Knee Actions Search in PubMed Search in NLM Catalog Add to Search,2013,20(2):96-99.

\section{Figures}
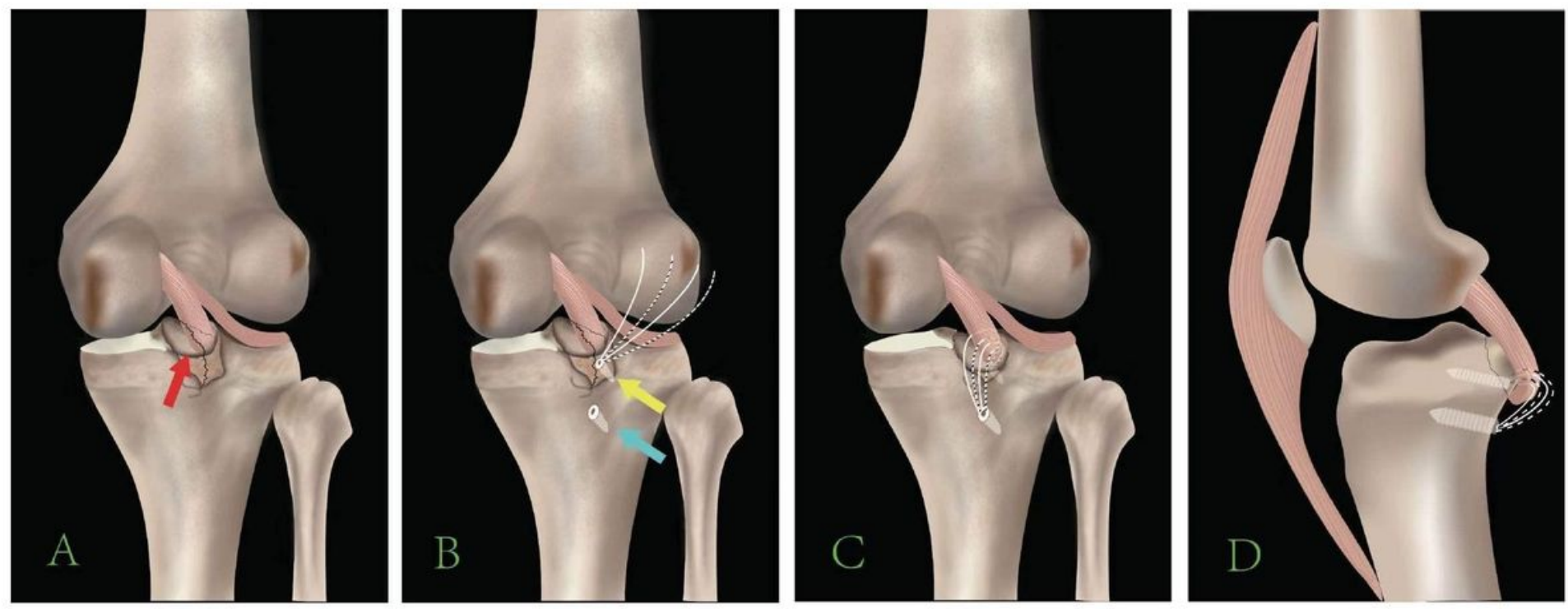

Figure 1

Schematic drawings of double row absorbable anchor bridge suture repair of PCL tibial avulsed fracture.

(A) showing the avulsed fragment from the tibia (red arrow) was used. (B) Approximate anatomic 


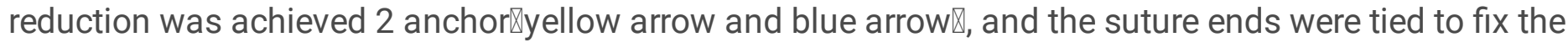
bone fragment. $₫ \mathrm{C} \bigotimes \mathrm{D} \bigotimes$ showing the position of the anchors in the coronal plane and in the sagittal plane.
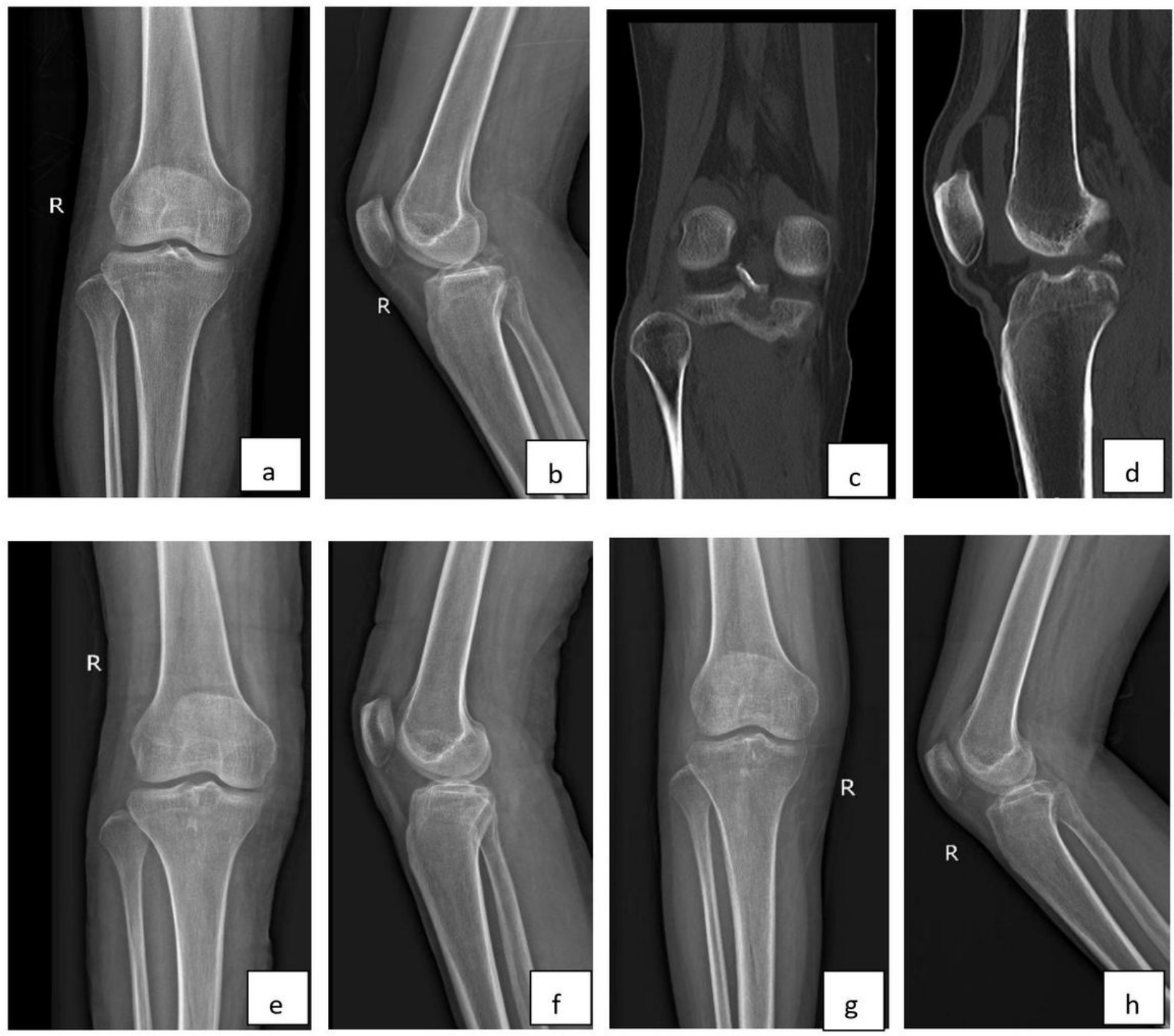

\section{Figure 2}

A 52-year-old female with PCL tibial avulsed fracture. Double row absorbable anchor bridge suture repair of PCL tibial avulsed fracture was performed. $(a, b)$ Preoperative anterior-posterior and lateral X-ray of the right knee showing the avulsed fragment from the tibia . (c,d) Preoperative omputed tomography scan showing the avulsed fragment of the tibia. (e,f) Anterior-posterior and lateral X-ray films of the knee after surgery showing anatomic reduction of fracture. $(\mathrm{g}, \mathrm{h})$ Anterior-posterior and lateral X-ray films of the knee operation 3 months showing good fracture healing. 

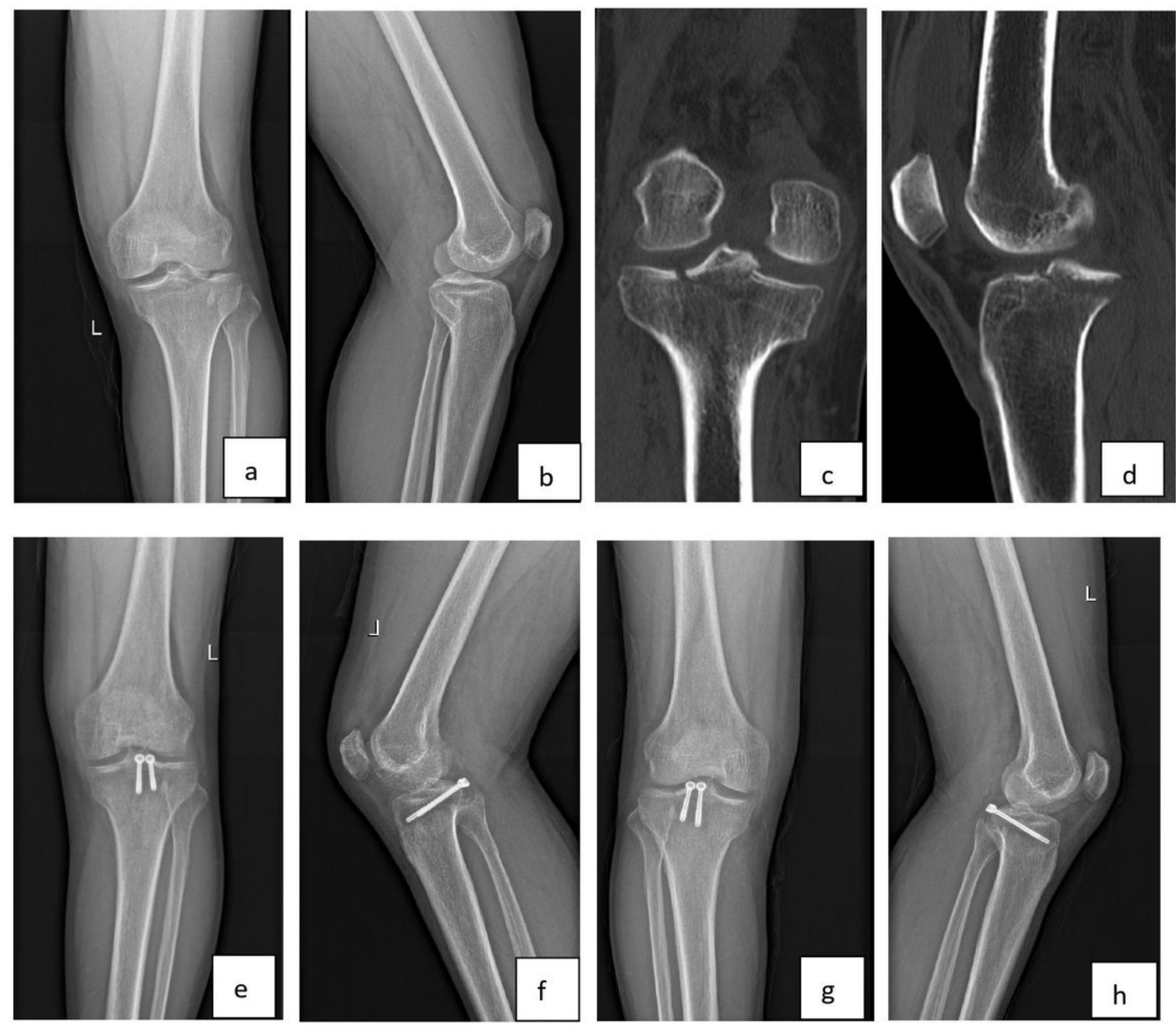

\section{Figure 3}

A 32-year-old male with PCL tibial avulsed fracture. hollow nails repair of PCL tibial avulsed fracture was performed. $(a, b)$ Preoperative anterior-posterior and lateral X-ray of the left knee showing the avulsed fragment from the tibia . (c,d) Preoperative omputed tomography scan showing the avulsed fragment of the tibia. $(e, f)$ Anterior-posterior and lateral X-ray films of the knee after surgery showing anatomic reduction of fracture. (g,h) Anterior-posterior and lateral X-ray films of the knee operation 3 months showing good fracture healing. 